\title{
White Matter Compromise in Veterans Exposed to Primary Blast Forces
}

\author{
Katherine H. Taber, PhD; Robin A. Hurley, MD; Courtney C. Haswell, MS; \\ Jared A. Rowland, PhD; Susan D. Hurt, PhD; Cory D. Lamar, MD; Rajendra A. Morey, MD
}

\begin{abstract}
Objective: Use diffusion tensor imaging to investigate white matter alterations associated with blast exposure with or without acute symptoms of traumatic brain injury (TBI). Participants: Forty-five veterans of the recent military conflicts included 23 exposed to primary blast without TBI symptoms, 6 having primary blast with mild TBI, and 16 unexposed to blast. Design: Cross-sectional case-control study. Main Measures: Neuropsychological testing and diffusion tensor imaging metrics that quantified the number of voxel clusters with altered fractional anisotropy (FA) radial diffusivity, and axial diffusivity, regardless of their spatial location. Results: Significantly lower FA and higher radial diffusivity were observed in veterans exposed to primary blast with and without mild TBI relative to blast-unexposed veterans. Voxel clusters of lower FA were spatially dispersed and heterogeneous across affected individuals. Conclusion: These results suggest that lack of clear TBI symptoms following primary blast exposure may not accurately reflect the extent of brain injury. If confirmed, our findings would argue for supplementing the established approach of making diagnoses based purely on clinical history and observable acute symptoms with novel neuroimaging-based diagnostic criteria that "look below the surface" for pathology. Key words: diffusion tensor imaging (DTI), diffusivity, fractional anisotropy, mild traumatic brain injury (mTBI), military veterans, primary blast, subconcussive blast exposure, white matter
\end{abstract}

A LTHOUGH EXPOSURE to explosive forces emanating from bombs and other devices is increasing among civilians and is common in veterans of recent

\footnotetext{
Autbor Affiliations: Mid-Atlantic Mental Illness Research Education and Clinical Center, Durbam, North Carolina (Drs Taber, Hurley, Rowland, Lamar, and Morey and Ms Haswell); Research and Education Service Line, W. G. (Bill) Hefner VA Medical Center, Salisbury, North Carolina (Drs Taber, Hurley, Rowland, Hurt, and Lamar); Division of Biomedical Sciences, Edward Via College of Osteopathic Medicine, Blacksburg, Virginia (Dr Taber); Departments of Physical Medicine and Rehabilitation (Dr Taber) and Psychiatry and Behavioral Sciences (Dr Hurley), Baylor College of Medicine, Houston, Texas; Departments of Psychiatry and Behavioral Medicine (Drs Hurley and Rowland), Radiology (Dr Hurley), Neurobiology and Anatomy (Dr Rowland), Wake Forest School of Medicine, Winston-Salem, North Carolina; Durbam VA Medical Center, Durham, North Carolina (Ms Haswell and Dr Morey); Duke-UNC Brain Imaging and Analysis Center (Ms Haswell and Dr Morey) and Department of Psychiatry and Behavioral Sciences (Dr Morey), Duke University, Durham, North Carolina.
}

This research was supported by a grant from the Department of Defense, Joint Improvised Explosive Device Defeat Organization (51467EGJDO), the Department of Veterans Health Affairs Rehabilitation Research and Development (RX000389-01), and with resources of the Mid-Atlantic Mental Illness Research, Education, and Clinical Center and W. G. (Bill) Hefner VA Medical Center.

The viewes expressed in this article are those of the authors and do not necessarily reflect the position or policy of the Department of Veterans Affairs, the Department of Defense, or the US government.

The authors declare no conflicts of interest.

Corresponding Author: Katherine H. Taber, PbD, Research Health Scientist, W. G. (Bill) Hefner VA Medical Center, Research and Education Service Line, Mailstop 11M, 1601 Brenner Ave, Salisbury, NC 28144 (Katherine.taber@va.gov).

DOI: $10.1097 /$ HTR.0000000000000030 military conflicts in Iraq and Afghanistan, relatively little is known about the consequences to the brain. ${ }^{1-8}$ Animal studies and computer modeling indicate that the blast wave has the potential to induce brain injury by different mechanism(s) than are present in nonpenetrating (closed head) traumatic brain injury (TBI) of more conventional origin, such as impact injury. ${ }^{2,5,7-9}$ This suggests that secondary injury and recovery processes may also differ. Recent studies have also raised other worrisome possibilities, including subconcussive effects and induction of chronic traumatic encephalopathy, making study in humans essential.

The severity of TBI is determined primarily by symptoms immediately following the event, such as altered sensorium, loss of consciousness, and presence/duration of posttraumatic amnesia. ${ }^{10}$ Most combat-related TBIs are classified as mild on the basis of symptoms at the time of injury (eg, dazed/confused/"saw stars," at most a short loss of consciousness or a brief period of amnesia). ${ }^{4,6,8,10}$ Most events involve a combination of primary blast and other forces, often described as "blast plus" or blast-related TBI., 5,7,8 Preliminary evidence suggests that early evolution of blast-related mild TBI may differ from other injury mechanisms. ${ }^{1,6}$ Differences in injury mechanism(s) and/or injury evolution make it essential to determine the effects in the human brain of exposure to primary blast. A case series and 2 case reports support the vulnerability of white matter (WM) regions to primary blast injury, indicated by small, spatially dispersed areas of abnormally low 
fractional anisotropy (FA) on diffusion tensor imaging (DTI). ${ }^{11-13}$

Diffusion tensor imaging is a type of magnetic resonance imaging that is sensitive to the diffusion of water in tissue and able to provide an indirect measure of WM integrity. ${ }^{14,15}$ Within gray matter, water diffuses at similar rates in all directions (isotropic). Within WM, microstructural barriers (eg, membranes, myelin, and neurofilaments) cause water to diffuse faster along the longitudinal axis of axons (anisotropic). Using measures such as FA to quantify directionality allows inferences to be made about the integrity of the underlying WM. Higher values of FA reflect greater directionality and suggest healthy WM, while loss of tissue integrity at the microscopic level (eg, shearing injury and demyelination) will manifest as reduced FA.

Adding to the complexity of the situation, evidence is accumulating that subconcussive blast exposure might injure the brain. ${ }^{16,17}$ A recent study in veterans reported a strong association between a history of exposure to blast and reduced first percentile FA that was independent of symptoms at the time of exposure indicative of mild TBI. ${ }^{16}$ There is also emerging evidence that subconcussive sports-related events are potentially injurious to the brain. ${ }^{18,19}$ A study comparing young concussionnaive athletes participating in a contact sport (soccer) and a noncontact sport (swimming) found multiple areas of significantly increased radial diffusivity $(R D)$ and axial diffusivity (AD) in the contact group. ${ }^{20} \mathrm{~A}$ study of amateur soccer players reported areas of reduced FA in players with higher number of headings, with evidence for a threshold effect. ${ }^{19}$ Similarly, studies comparing postseason with preseason DTI in young athletes (high school, college) participating in contact sports (ice hockey, football) found multiple areas with significantly altered FA and mean diffusivity postseason in the absence of concussive events during the playing season. ${ }^{21,22}$ Decrements in neurocognitive and functional imaging measures have also been reported in young athletes following multiple in-game subconcussive hits. ${ }^{23,24}$ Finally, autopsy-confirmed chronic traumatic encephalopathy was reported in a college football player with no reported concussions. ${ }^{25}$

The purpose of this study was to assess WM integrity in previously deployed veterans with a history of primary blast exposure with and without clinical symptoms of mild TBI at the time of exposure. We hypothesized that the effects of primary blast exposure would be detected as reduced WM integrity on DTI and that the magnitude of changes would be associated with the intensity of exposures. Furthermore, we predicted, as with mild TBI due to other types of forces, the compromise to WM integrity would be diffuse and widely dispersed with clear interindividual spatial heterogeneity. Accordingly, we predicted that cognitive performance deficits would be most prominent for cognitive tasks requiring widely distributed neural systems such as working memory and executive function.

\section{METHODS}

This study was conducted at the Department of Veterans Affairs Mid-Atlantic Mental Illness Research, Education and Clinical Center. The welfare of human subjects was protected. Participants provided informed consent to take part in research procedures approved by the institutional review board at the W. G. "Bill" Hefner VA Medical Center, Salisbury, North Carolina.

\section{Participants}

Study eligibility of veterans who served since September 11, 2001, was determined by review of medical records and a screening interview. Exclusion criteria include a predeployment history of neuropsychiatric, neuropsychological, and/or neurological symptoms, including head trauma (other than mild concussion, indicated by not more than momentary loss of consciousness), seizures, strokes, prior neurosurgery, evidence of mental retardation, neurological impairments, substance dependence, Axis I psychiatric disorders; exposure to conditions during or following deployment likely to result in a TBI due to forces other than primary blast (eg, impact injury); and presence of shrapnel, metallic implants, devices, or conditions contraindicating neuroimaging. There was one subject with inadequate quality of DTI images from each of the 3 groups. Thus, data were analyzed from those who were (i) unexposed $(n=16)$, reported no exposure during deployment to any conditions likely to result in a TBI; (ii) primary blast-exposed $(n=23)$, reported exposure only to primary blast forces, and either no symptoms at the time or symptoms that did not meet criteria for mild TBI; (iii) with primary blast-induced TBI $(n=6)$, reported exposure only to primary blast forces, symptoms at the time consistent with mild TBI according to established criteria, ${ }^{10}$ and present symptoms consistent with residual mild TBI as determined by an experienced Veterans Affairs clinician.

\section{Clinical testing}

Psychiatric status was determined by administration of the Structured Clinical Interview for Diagnostic and Statistical Manual of Mental Disorders (Fourth Edition) Axis I disorders (SCID) by an experienced clinical psychologist. Computer-based neurocognitive testing used the following 8 tests from the Cambridge Neuropsychological Test Automated Battery (CANTABeclipse v3.0, Cambridge Cognition Ltd, 
Cambridge, United Kingdom): Reaction Time (cognitive and motor processing speed), Delayed Matching to Sample (visual working memory), Spatial Working Memory (SWM; visuospatial working memory), Stockings of Cambridge (visuospatial working memory and sequence planning), Intra-Extra Dimensional Shift (IED; learning by inferring rules and setshifting), Cambridge Gambling Task (decision-making and risk-taking behavior outside a learning context), Affective Go-NoGo (information processing bias and inhibitory control for positive and negative), and Rapid Visual Information Processing (visual working memory and sustained attention).

\section{Image acquisition}

Images were acquired on a General Electric Signa HDxt 1.5 Tesla (GE Healthcare, Milwaukee, WI) scanner with an 8-channel receive coil. All participants underwent DTI with $2 \times 2 \times 6-\mathrm{mm}$ voxel size, $128 \times 128 \times 24$ matrix automatically resampled to $1 \times 1 \times 6-\mathrm{mm}$ voxel size, field of view $240 \times 192-\mathrm{mm}$, flip angle $90^{\circ}$, time of repeat $=8500 \mathrm{~ms}$, time of echo $=107 \mathrm{~ms}, 1$ average, 25 noncollinear directions (diffusion gradients), sensitivity encoding factor $=1$, nonzero $b$ value $=1000 \mathrm{~s} / \mathrm{mm}^{2}$, and scanning time $=12^{\prime} 30^{\prime \prime}$. All images were visually inspected for the purpose of quality assurance.

\section{Preprocessing of diffusion imaging data}

Preprocessing was carried out using the FMRIB Diffusion Tool Box (FMRIB Centre, Oxford University, Oxford, United Kingdom) to remove eddy current distortions caused by the stretching and shearing of diffusion weighted images by gradient coils, and to correct for simple head motion. Fractional anisotropy, the primary measure acquired from the DTI data is a scalar metric describing the WM integrity, was calculated from the orientational coherence of the diffusion compartments within a voxel. The analytic approach was based on skeleton voxels identified by tract-based spatial statistics (TBSS). All subjects in the sample were coregistered using a method that ensured WM alignment using an intermediate degrees of freedom, nonlinear registration to a $1 \times 1 \times 1-\mathrm{mm}$ template (FMRIB58_FA) of male and female subjects aged 20 to 50 years (http://www.fmrib.ox.ac.uk/fsl/). These normalized resampled images were averaged to generate a mean FA image. In TBSS, a mean FA skeleton was created as lines and surfaces that pass through the centers of WM tracts in the mean FA image. After thresholding the skeleton to exclude low FA values $(<0.2)$ indicative of non-WM, each subject's aligned FA image was projected onto the mean FA skeleton. A voxelwise whole-brain approach was used instead of a region-of-interest approach: computing statistics for each voxel independently.

\section{Significance testing for abnormal FA}

Assumptions about the distribution of DTI data (eg, Gaussianity) were avoided by using permutation testing to make inferences about group differences and associations with clinical regressor variables from a samplespecific probability distribution of means. This nonparametric analysis was conducted using randomise (FMRIB Centre, University of Oxford, Oxford, United Kingdom), an implementation of permutation testing with covariates (clinical regressors) for whole-brain voxelwise analyses, to establish a significance level for every skeleton voxel from a distribution generated by 5000 permutations of the group label. For details about permutation testing with regressors, see Kennedy ${ }^{26}$ or Anderson and Robinson. ${ }^{27}$

\section{Clinical regressors}

Four covariates were included in the initial wholebrain voxelwise analysis of FA: age, premilitary exposure to mild concussion, posttraumatic stress disorder (PTSD) diagnosis, and alcohol use score, which was compiled from assessing participants' amount of alcohol consumption, level of psychological impact, level of physiological impact, and symptoms of withdrawal. Intermediate results showed that the alcohol use score and premilitary mild concussions were poorly correlated with FA $\left(P_{\min }>.9\right)$. Therefore, only significant and trend-level significant covariates of age and PTSD diagnosis were retained in the whole-brain analyses.

\section{Correction for multiple comparisons}

The results of permutation testing were followed up with correction for multiple comparisons by using threshold-free cluster enhancement as an alternative to the overly conservative corrections, such as Bonferroni, that have poor control of type II error. ${ }^{28}$ Rather than requiring the selection of an arbitrary initial clustering threshold for subsequent computation of $P$ values based on Gaussian Random Field theory (cluster-level correction), threshold-free cluster enhancement accounts for "cluster-like local spatial support," that is, intensity and extent of the test statistic. Recommended (default) threshold-free cluster enhancement parameters for cluster height $(H=2)$, cluster extent $(E=1)$, and cluster connectivity $(C=26)$ were used with randomise.

\section{Visualization of results}

The significance maps $(P<.05$; corrected $)$ were superimposed on the normalized group skeleton of the FMRIB58_FA template. The TBSS-fill feature was applied to all significance maps to enhance the visualization of TBSS results as commonly used in similar 
studies. ${ }^{29-31}$ Tracts are reported according to standard nomenclature. ${ }^{32}$

\section{Statistical analyses of $\mathrm{z}$ score voxel clusters}

Preprocessing of DTI data was performed as described earlier. We compared the number of voxels with abnormal DTI metrics with minimal spatial constraints among participants to determine the possible effect of primary blast on small regions of WM. The individual subject and the reference group were registered to the template. Each participant's DTI metric map was compared with images of the mean and standard deviation of the reference group of 16 unexposed control participants. An individual $z$ score map was created for each participant in the primary blast-induced TBI and the primary blastexposed group that was thresholded at $z=-2.0$ or less for $\mathrm{FA}$, and $z=2.0$ or more for $\mathrm{RD}$ and AD. Given that the $z$ statistic is valid only for normally distributed data, the $z$ map was masked with reference-group values that were normally distributed as assessed by the Lilliefors ${ }^{33}$ test. Overall, this procedure was designed to identify voxels with values in the extremes of the healthy distribution rather than voxels deemed statistically significantly different than the healthy mean. ${ }^{34,35}$

\section{Group statistical analyses of $\mathbf{z}$ score voxel clusters}

We adopted an approach similar to White et $\mathrm{a}^{35}$ for analyzing spatially heterogeneous changes in DTI metrics among subjects and groups. Summary statistics for the primary blast-exposed and primary blast-induced TBI group were computed from the individual subject comparisons to the reference group in the previous step. Group statistics for the average number of voxel clusters with abnormal metrics (FA less than $z=-2$, RD or $\mathrm{AD}$ more than $z=2$ ) were binned according to size in increments of 25 voxels. Voxel clusters were classified into 3 sizes: small (25-49), medium (50-74), and large (75-100). Very small voxel clusters (fewer than 25 voxels) and voxel clusters with extremely low cluster counts owing to their large size (exceeding 100 voxels) were excluded from the analyses to minimize spurious results (type I error). The number of small, medium, and large voxel clusters for each DTI metric was tabulated for each participant, as well as the mean and standard deviation for the primary blast-induced TBI, primary blastexposed, and the unexposed control groups. Histogram plots were generated for the number of voxel clusters of each size in the 3 participant groups. We used repeatedmeasures generalized linear model to quantify differences between the primary blast-exposed and primary blast-induced TBI groups compared with the unexposed control group. Repeated measures were counts of small, medium, and large voxel clusters. The generalized linear model included covariates for PTSD diagnosis, ${ }^{16,36}$ diagnosis of alcohol use disorders, ${ }^{37}$ premilitary exposure to mild concussion, and age ${ }^{38}$ given prior reports of association with FA. However, alcohol use disorder, premilitary exposure to mild concussion, and PTSD diagnosis were poorly correlated with FA in intermediate results (data not shown). Therefore, age was the only covariate retained in our primary DTI analyses. Finally, we examined the spatial distribution of FA voxel clusters by creating a whole-brain voxelwise histogram of the number of voxel clusters exceeding 25 voxels ( $\geq$ small voxel cluster) in the each of the 2 affected groups. The histogram map was produced by indicating the ordinal count of the number of subjects for which a given voxel was part of a voxel cluster.

\section{Correspondence between DTI findings and cognitive performance}

Principal component analysis was conducted on the 9-voxel cluster variables (AD, RD, and FA for small, medium, and large voxel clusters, respectively) to reduce multicollinearity among these variables for use in subsequent hierarchical linear regression models. These 9 variables loaded onto 2 components (all variables loaded at 0.93 or greater) using direct-oblimin rotation to explain $91 \%$ of the underlying variance. The first component included all $R D$ and $A D$ variables, and the second component included all FA variables. Hierarchical linear regression analyses were conducted to examine how the 2 DTI component scores related to performance on cognitive testing beyond possibly confounding demographic variables (age, education, and race) known to relate to cognitive performance, as well as the number of PTSD and major depression (MDD) symptoms reported during the SCID. Demographic variables were entered as step 1, PTSD and MDD symptoms as step 2, followed by the 2 DTI component scores as step 3 . Neuropsychological tests, selected on the basis of cognitive constructs requiring an anatomically distributed network of regions given the widespread pattern of voxel clusters, included Delayed Matching to Sample (percent correct), IED (stages completed, percent preshift errors, and percent postshift errors), Cambridge Gambling Task (quality of decision-making and risk-taking), Stockings of Cambridge (problems completed in minimum moves and the average number of moves), RTI (simple reaction time), and SWM total errors.

\section{RESULTS}

\section{Clinical variables}

Detailed demographic and clinical characteristics of the 3 participant groups are provided in Table 1 . 
TABLE 1 Demographic and clinical characteristics of participants

\begin{tabular}{|c|c|c|c|c|c|}
\hline Characteristic & $\begin{array}{c}\text { Blast } \\
\text { mild TBI } \\
(n=6)\end{array}$ & $\begin{array}{c}\text { Blast } \\
\text { exposed } \\
(n=23)\end{array}$ & $\begin{array}{l}\text { Unexposed } \\
\text { control } \\
(n=16)\end{array}$ & $\begin{array}{c}\text { Group comparison } \\
\text { (mild TBI vs } \\
\text { control) }\end{array}$ & $\begin{array}{c}\text { Group comparison } \\
\text { (exposed vs } \\
\text { control) }\end{array}$ \\
\hline Age (SD), y & $35.8(8.7)$ & $35.8(7.4)$ & $37.3(11.5)$ & $t_{20}=0.28, P<.78$ & $t_{37}=0.49, P=.63$ \\
\hline Sex, females, $n(\%)$ & $0(0)$ & $5(21.7)$ & $4(25.0)$ & $\chi^{2} 1=1.8, P<.18$ & $\chi^{2}{ }_{1}=0.06, P=.81$ \\
\hline $\begin{array}{l}\text { Handedness, right-handed, } \\
n(\%)\end{array}$ & $6(100.0)$ & 21 (91.3) & 15 (93.8) & $\chi^{2}{ }_{1}=0.39, P<.53$ & $\chi^{2}{ }_{1}=0.08, P<.78$ \\
\hline Race, white subjects, $n(\%)$ & 5 (83.3) & $15(65.2)$ & $12(75.0)$ & $\chi^{2}{ }_{1}=0.01, P<.91$ & $\chi^{2}{ }_{1}=1.2, P<.27$ \\
\hline Education (SD), y & $13.8(0.98)$ & $14.3(2.2)$ & $13.7(2.0)$ & $t_{20}=-0.17, P<.87$ & $t_{37}=-0.83, P<.41$ \\
\hline $\begin{array}{l}\text { Prior exposure to concussion, } \\
n(\%)\end{array}$ & $0(0)$ & $3(13.0)$ & $2(12.5)$ & $\chi^{2} 1=0.87, P<.35$ & $\chi^{2}{ }_{1}=0.002, P=.96$ \\
\hline $\begin{array}{l}\text { SCID PTSD symptoms mean } \\
\text { (SD) }\end{array}$ & $14.7(1.4)$ & $6.4(5.6)$ & $3.8(4.8)$ & $t_{20}=-5.4, P<.001$ & $t_{37}=-1.5, P<.14$ \\
\hline Diagnosis of PTSD, $n(\%)$ & $6(100)$ & $7(30.0)$ & $3(18.8)$ & $\chi^{2}{ }_{1}=11.9, P<.001$ & $\chi^{2}{ }_{1}=0.68, P=.41$ \\
\hline $\begin{array}{l}\text { Diagnosis of anxiety disorder, } \\
n(\%)\end{array}$ & $6(100)$ & $9(39.1)$ & $4(25.0)$ & $\chi^{2}{ }_{1}=7.1, P=.008$ & $\chi^{2}{ }_{1}=0.85, P=.36$ \\
\hline Alcohol use disorder, $n(\%)$ & $3(50)$ & $9(39.1)$ & $1(6.3)$ & $\chi^{2}{ }_{1}=0.23, P=.63$ & $\chi^{2}{ }_{1}=5.4, P=.02$ \\
\hline $\begin{array}{l}\text { Diagnosis of drug } \\
\text { dependence, } n(\%)\end{array}$ & $3(50)$ & $6(26.1)$ & $3(18.8)$ & $\chi^{2}{ }_{1}=1.2, P=.26$ & $\chi^{2}{ }_{1}=0.29, P=.58$ \\
\hline
\end{tabular}

Abbreviation: PTSD, posttraumatic stress disorder.

\section{Voxelwise whole-brain analyses}

A whole-brain voxelwise comparison of FA between the blast-exposed and the blast-unexposed control group was covaried for age and diagnosis of PTSD assessed with the SCID (see Figure 1). These results show significantly lower FA in the primary blast-exposed group compared with the unexposed group in diffuse cortical and subcortical tracts in the right hemisphere, including the forceps major, superior and inferior longitudinal fasciculus, anterior thalamic radiations, inferior frontooccipital fasciculus, and the corticospinal tract $(P<.05$; corrected). The left hemisphere did not show differences in FA between the primary blast-exposed group and the unexposed control group $(P<.05$; corrected $)$. At a reduced significance level $(P<.15$; corrected $)$, the left hemisphere showed group-differences in FA that were closer in magnitude and extent to the finding in the right hemisphere. The inconsistent findings between the left and right hemisphere highlighted spatial heterogeneity among primary blast-exposed individuals further motivating an alternate approach for assessing WM injury independent of spatial location. There were no significant differences with this technique comparing the primary mild TBI group with the unexposed group or to the primary blast-exposed group.

\section{Spatially independent assessment of FA reductions}

The magnitude of WM injury as measured by FA that was independent of spatial location is shown for the primary blast-induced TBI, primary blast-exposed, and the unexposed control groups in Figure 2. There was a significant main effect of group on the number of small, medium, and large voxel clusters (repeated measures) $\left(F_{2,42}=4.4, P<.02\right)$. The unexposed control group had significantly fewer voxel clusters than the primary blastinduced TBI group $(P=.009)$ and the primary blastexposed group $(P=.036)$; the primary blast-induced TBI and primary blast-exposed groups were not significantly different $(P=.2)$. Thus, on the basis of the number of voxel clusters, the primary blast-exposed group resembled the primary blast-induced TBI group when considered in relation to the unexposed control group (Fig 2). Age was significantly correlated with number of voxel clusters $\left(F_{1,41}=7.3, P=.01\right)$. Also as expected, there was a main effect of volume size on the number of voxel clusters $\left(F_{2,82}=19.4, P<.0001\right)$, with significantly fewer large voxel clusters than medium voxel clusters, and fewer medium voxel clusters than the small voxel clusters. Finally, there was an interaction of group $\times$ volume size $\left(F_{4,82}=3.8, P=.007\right)$ where the small voxel clusters had greater between-group differences than the medium and larger volume sizes (Fig 2).

\section{Spatial distribution of FA reductions}

The histogram map of voxel clusters larger than 25 voxels (see Figure 3 ) demonstrates that voxel clusters in both the primary blast-induced TBI and primary blastexposed group were distributed heterogeneously both within and across subjects, which was consistent with our second hypothesis. The histogram map shows that for the preponderance of locations (voxels), there were 


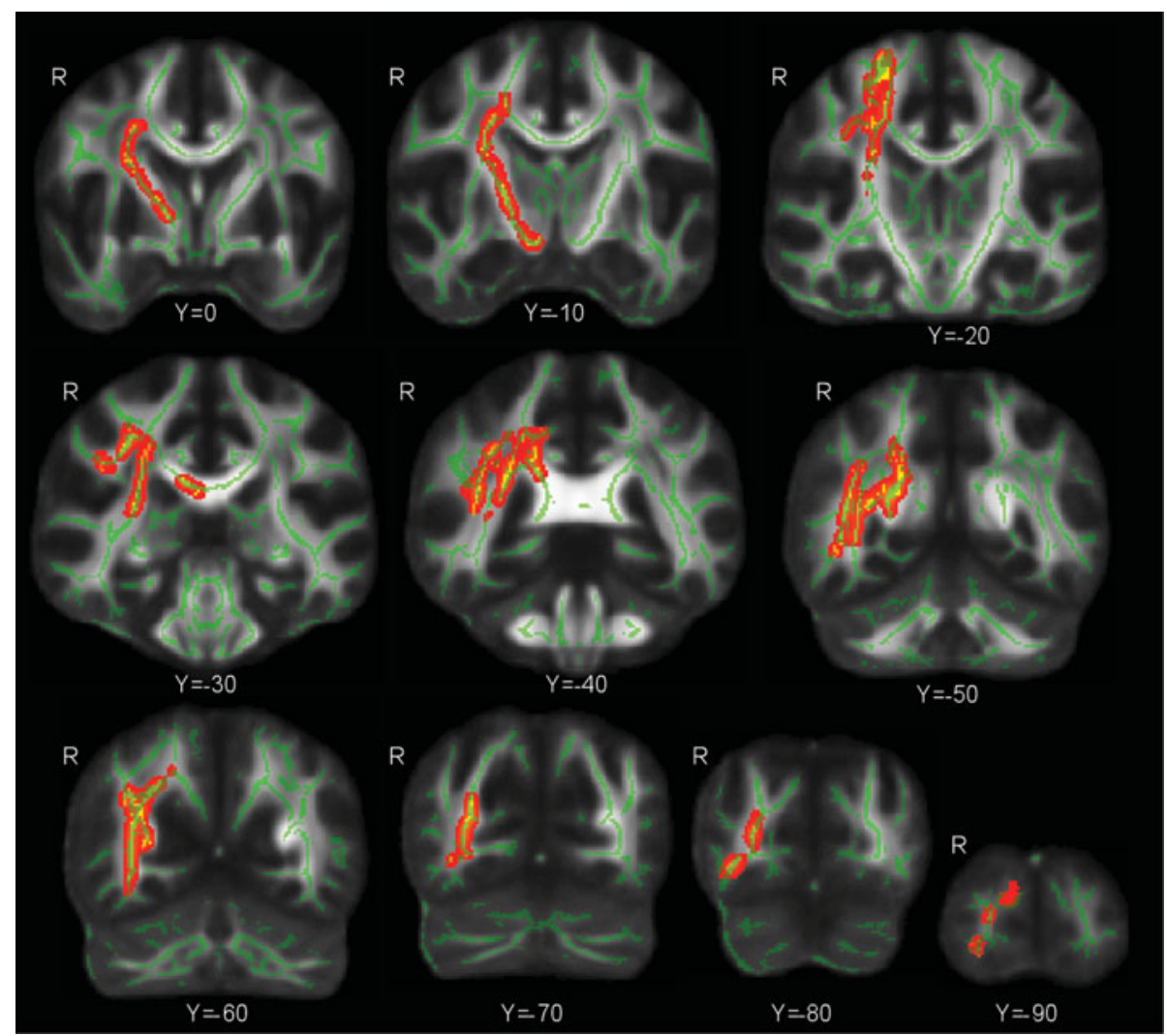

Figure 1. Whole-brain voxelwise comparison of FA between the blast-exposed group and the blast-unexposed control group was covaried for age and posttraumatic stress disorder diagnosis. These results show significantly lower FA in the blast-exposed group than that in the blast-unexposed group in diffuse cortical and subcortical tracts based in the right hemisphere, including the forceps major, superior and inferior longitudinal fasciculus, anterior thalamic radiations, inferior fronto-occipital fasciculus, and the corticospinal tract $(P<.05$; corrected). This figure is available in color in the article on the journal website, www.headtraumarehab.com, and the iPad.

voxel clusters present in only 1 participant, a few locations where voxels clusters were present in 2 or 3 participants, and rare cases of locations where voxel clusters were present in 4 to 7 participants.

\section{Spatial distribution of radial and AD elevations}

The magnitude of WM injury, measured as elevations in $\mathrm{RD}$ that were independent of spatial location, is shown for primary blast-induced TBI, primary blastexposed, and the unexposed control groups in Figure 4. There was a significant main effect of group on the number of small, medium, and large $\mathrm{RD}$ voxel clusters (repeated measures) $\left(F_{2,42}=3.7, P=.034\right)$. The unexposed control group had significantly fewer $\mathrm{RD}$ voxel clusters than the primary blast-induced TBI group $(P=$ $.03)$ and the primary blast-exposed group $(P=.025)$, with no significant difference between the primary blastinduced TBI and primary blast-exposed groups $(P=.5)$. Age was not significantly correlated with the number of voxel clusters $\left(F_{1,41}=.005, P=.9\right)$. There was no main effect of size on the number of $R D$ voxel clusters $\left(F_{2,82}=\right.$ $2.2, P=.12)$. Finally, there was an interaction of group $\times$ volume size $\left(F_{4,82}=4.2, P=.004\right)$ where small voxel clusters had greater between-group differences than the medium and large voxel clusters (Fig 4).

The magnitude of WM injury, measured as elevations in $\mathrm{AD}$ that were independent of spatial location, is shown for the primary blast-induced TBI, primary blast-exposed, and the unexposed control groups in Figure 5. There was a nonsignificant main effect of group on the number of small, medium, and large $\mathrm{AD}$ voxel clusters (repeated measures) $\left(F_{2,42}=2.0, P=.15\right)$. Age was not significantly correlated with the number of voxel clusters $\left(F_{1,41}=2.4, P=.13\right)$. There was a main effect of size on the number of $A D$ voxel clusters $\left(F_{2,82}=8.7, P<\right.$ $.0001)$, with significantly fewer large than medium voxel clusters, and fewer medium voxel clusters than small.

\section{Neuropsychological measures}

Hierarchical linear regression models, including age, race, education, PTSD symptoms, MDD symptoms, and DTI metric components, significantly predicted the IED shift number of stages completed $\left(F_{7,37}=2.4, P<.04\right)$ and postshift percent errors $\left(F_{7,37}=2.8, P<.02\right)$; simple 


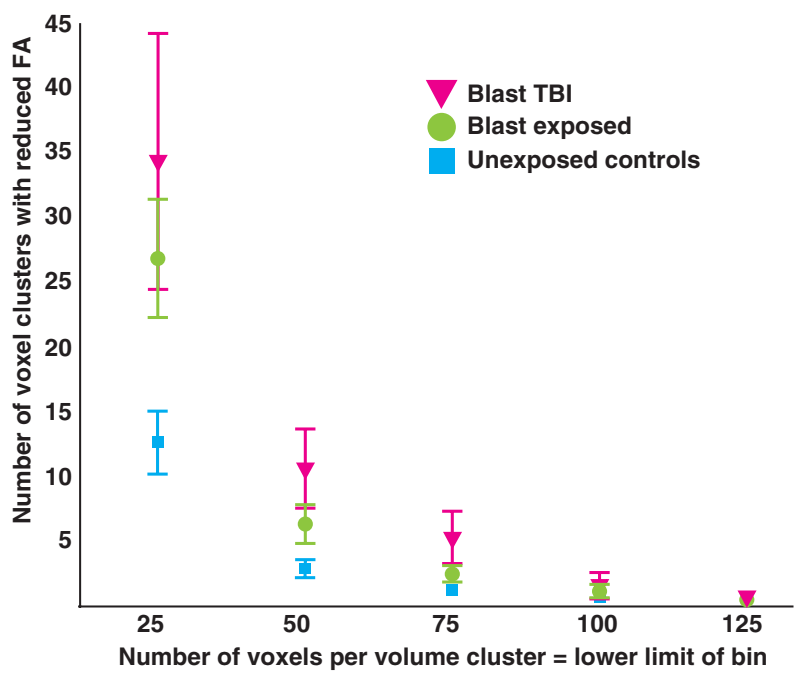

Figure 2. There was a significant main effect of group $\left(F_{2,42}=\right.$ $4.4, P<.02)$ on the number of small ( $25-50$ voxels), medium (50-75 voxels), and large (75-100 voxels) voxel clusters, which were defined by low FA values $(z<-2)$. The unexposed control group had significantly fewer voxel clusters than the blast TBI group $(P=.009)$ and the blast-exposed group $(P$ $=.036)$. On the basis of the number of voxel clusters, the blast-exposed group resembled the blast TBI group when considered in relation to the unexposed control group. FA indicates fractional anisotropy; TBI, traumatic brain injury. This figure is available in color in the article on the journal website, www.headtraumarehab.com, and the iPad.

reaction time $\left(F_{7,37}=5.8, P<.001\right)$; as well as the number of errors on the SWM test $\left(F_{7,37}=2.3, P<.05\right)$. The addition of DTI metric components significantly improved the IED postshift percent errors $(P<.05)$ and simple reaction time $(P<.05)$ but not the SWM $(P<.17)$ or IED stages complete $(P<.08)$ models. At the individual level, the FA component was a significant predictor of simple reaction time $(\beta=-.340, P<.02)$ and SWM errors $(\beta=-.343, P<.04)$, each in the expected direction. The diffusivity component was a significant predictor of IED postshift percent error $(\beta=$ $.348, P<.02)$ in the expected direction.

\section{DISCUSSION}

The major finding of this study is that altered WM metrics were present in veterans with a remote ( $>1$ year) history of exposure to primary blast forces both with (primary blast-induced TBI) and without (primary blastexposed) self-report of symptoms at the time of the event consistent with criteria for mild TBI. A spatially diffuse distribution of abnormal voxel clusters was present in both groups consistent with the anatomically heterogeneous and individually variable nature of injury identified in both imaging and neuropathological studies of mild TBI. ${ }^{39,40}$ The analytic approach allowing for heterogeneity in the location of altered metrics $(z$ score analysis) was more sensitive than the conventional whole-brain voxelwise method (TBSS) that relies on consistency in location. Although the TBSS analysis suggested that WM vulnerability was limited to the right parietal lobe in the primary blast-exposed group and left parietal lobe in the primary blast-induced TBI group, the $z$ score voxel cluster analysis demonstrated that the WM vulnerability was far more extensive. Diagnosis of PTSD was not correlated with WM injury, using either the spatially independent or conventional wholebrain analysis methods. The $z$ score approach did not indicate heightened vulnerability of any specific WM locations.

The presence of voxel clusters with abnormal DTI metrics in both the primary blast-exposed and primary blast-induced TBI groups is open to multiple interpretations. The challenges of obtaining accurate self-report of symptoms at a considerable time after the occurrence of combat-related events is well recognized. Thus, it is possible that the primary blast-exposed group included individuals who had experienced a more severe exposure than their memory of symptoms would indicate. Another possible explanation for relatively similar levels of WM compromise in the primary blast-exposed and the primary blast-induced TBI groups could be the focus of established criteria for mild TBI on altered sensorium. It stands to reason that the level and nature of symptoms experienced relates to the level of disruption of brain regions supporting specific functions. For instance, sufficient injury to the reticular activating system can produce altered consciousness, ${ }^{41}$ but a similar degree of injury to a cortical region might not produce overt symptoms. The possibility that primary blast forces may induce injury without causing clear alteration in consciousness is supported by studies reporting evidence for subconcussive effects of low level blast exposures and blunt force impacts in sports. The possibility that acute symptoms following exposure to primary blast may not accurately reflect injury state is also supported by a study in an urban trauma center reporting that almost one-third (10/32) of patients presenting with blast-induced head injury (none due to terrorist activity) received a delayed diagnosis of mild TBI following development of altered mental status and/or new-onset neuroimaging findings later in the hospital course. ${ }^{1}$

The widely distributed areas of loss of WM integrity is striking, and consistent with previous findings in mild TBI due to other injury mechanisms, confirming our hypothesis. ${ }^{39,40}$ Both nonblast and blast injury lead to changes at the cellular level via several biochemical pathways, including free radical generation, disruption of calcium homeostasis, and release of inflammatory mediators. ${ }^{2,5,8,39}$ Earlier models of impact TBI emphasizing focal or multifocal injury evolving from a pericontusional process have been supplanted with 

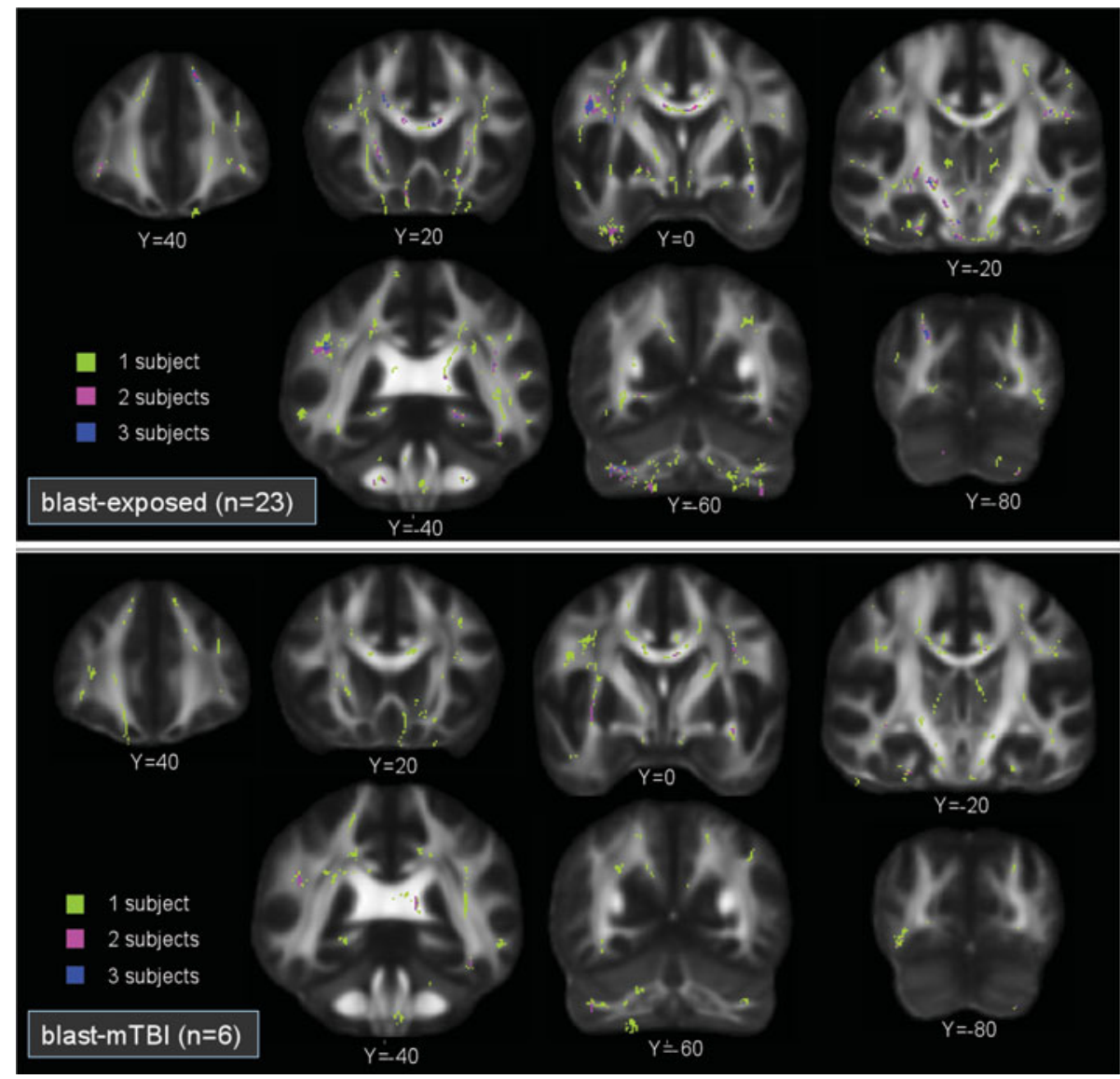

Figure 3. A whole-brain voxelwise histogram of voxel clusters more than 25 voxels in size demonstrates that voxel clusters in blast-exposed (top panel) and blast TBI (lower panel) groups were distributed heterogeneously both within and across subjects. The voxelwise histogram overlay shows that for most locations (voxels), there were voxel clusters present in only 1 participant (lime green). There were a few locations where voxel clusters were present in 2 (violet) or 3 (dark blue) participants. Only rarely were locations present in 4 to 7 subjects (data not shown). mTBI indicates mild traumatic brain injury. This figure is available in color in the article on the journal website, www.headtraumarehab.com, and the iPad.

a model that also includes diffuse mechanical forces of injury that may trigger noncontusion cell death cascades. ${ }^{42,43}$ The current hypotheses for primary blast-induced neurotrauma include mechanical forces, vasospasm, production of nitric oxide synthase, and glial activation, as well as immune-mediated and/or glutamate-mediated cell damage that results in apoptosis and necrosis of glial cells responsible for maintaining homeostasis, formation of myelin, and support of neurons. ${ }^{2,5,8}$ Our results appear to be consistent with loss of WM integrity that may be proximally explained by factors associated with the magnitude of the mechanical forces, but its diffuse pattern is also consistent with evidence of injury from downstream cascade of neurochemical and neurotoxic processes. ${ }^{42,43}$

Our result showed significant elevation of both $\mathrm{RD}$ and $\mathrm{AD}$ in the primary blast-exposed and primary blastinduced TBI groups compared with the control group. While it is tempting to interpret increased $\mathrm{RD}$ as suggestive of myelin injury and increased $\mathrm{AD}$ as suggestive of axonal injury, ${ }^{44}$ such conclusions must be made with caution. ${ }^{45,46}$ Simulations ${ }^{46}$ show that a change in $\mathrm{RD}$ can cause a fictitious change in $\mathrm{AD}$ and vice versa, particularly in voxels characterized by crossing fibers. Other confounding factors include partial volume effects from the low anisotropy, characteristic of gray matter, and when the diffusion ellipsoid is oblate, indicating the principal eigenvector has a large cone of uncertainty. ${ }^{47}$ Given that a typical DTI imaging voxel contains thousands of fibers, ${ }^{48}$ researchers are generally discouraged from interpreting changes in $\mathrm{AD}$ and $\mathrm{RD}$ on the basis of the underlying tissue structure. ${ }^{46}$

The neuropsychological effects of subconcussive blast are not well known. We observed a relationship between WM compromise (FA voxel clusters) and tests of simple reaction time and set shifting (an executive function subserved by widely distributed neural systems in the dorsolateral prefrontal, parietal, orbitofrontal, and anterior cingulate cortices) that was present even when controlling for other possibly confounding variables (age, race, 


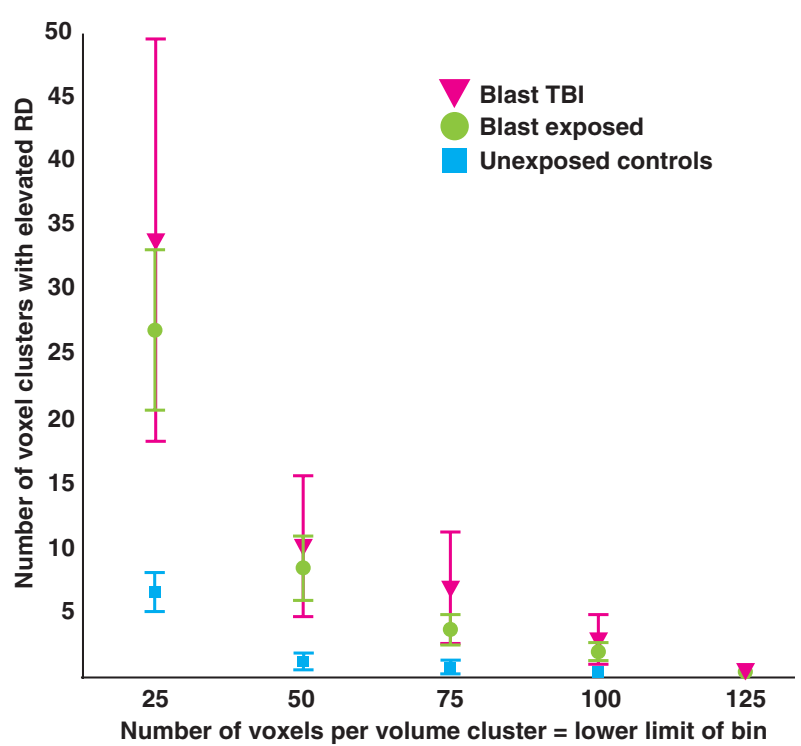

Figure 4. There was a significant main effect of group $\left(F_{2,42}=\right.$ $3.7, P=.034)$ on the number of small (25-50 voxel), medium (50-75 voxel), and large (75-100 voxel) voxel clusters, which were defined by high radial diffusivity $(z>2)$. The blastunexposed control group had significantly fewer voxel clusters than the blast TBI group $(P=.03)$ and the blast-exposed group $(P=.025)$. On the basis of the number of voxel clusters, the blast-exposed group resembled the blast TBI group when considered in relation to the blast-unexposed control group. $\mathrm{RD}$ indicates radial diffusivity; TBI, traumatic brain injury. This figure is available in color in the article on the journal website, www.headtraumarehab.com, and the iPad.

education, and PTSD and MDD symptoms). However, DTI metrics were not related to performance on several other cognitive tests, including other tests of executive functioning, such as decision-making, planning, and organization. Similarly, 2 recent studies have also reported altered DTI metrics associated with presence of functional deficits (sleep disturbances or executive dysfunction) in patients with mild TBI. ${ }^{49,50}$ Prior evidence of cognitive performance changes associated with subconcussive blast exposure is largely missing from the literature. Reports of pre- and postseason WM alteration from subconcussive sports injuries have shown decrements in visual motor speed and reaction time, ${ }^{21}$ whereas other studies of sports-related subconcussive exposure have not reported cognitive changes. ${ }^{20,22}$ Deficits related to WM compromise detected by DTI may manifest later in life when the effects of aging erode the benefits of neural redundancy and compensatory processes. ${ }^{51,52}$

\section{Caveats and limitations}

A few limitations deserve mention. The sample size of the primary blast-induced TBI group is small. When exposures occur in a battlefield situation, it can be extremely difficult to confidently identify cases of only

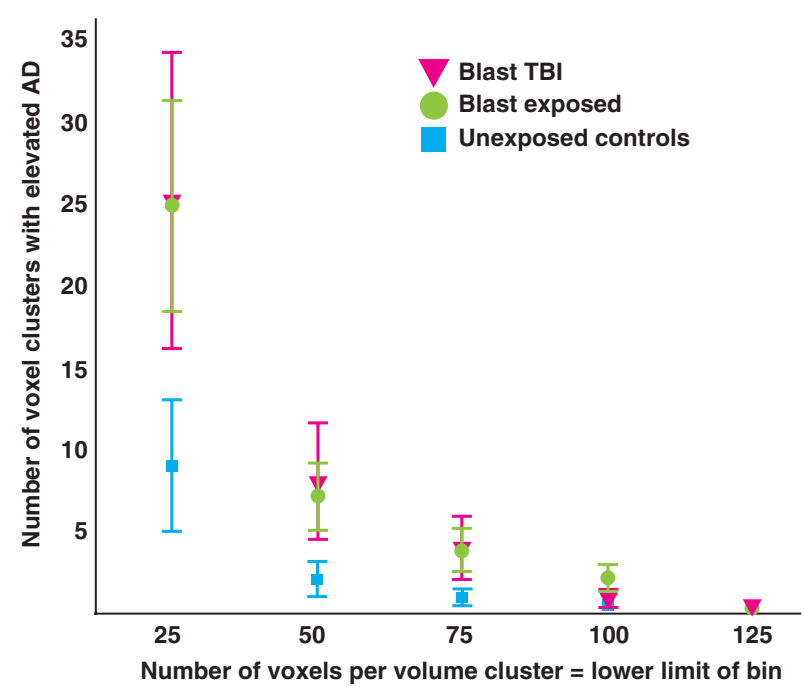

Figure 5. There was a nonsignificant main effect of group $\left(F_{2,42}=2.0, P=.15\right)$ on the number of small (25-50 voxel), medium (50-75 voxel), and large (75-100 voxel) voxel clusters, which were defined by voxels with high axial diffusivity values $(z>2)$. AD indicates axial diffusivity; TBI, traumatic brain injury. This figure is available in color in the article on the journal website, www.headtraumarehab.com, and the iPad.

primary blast injury. Moreover, isolated primary blast injury without secondary or tertiary injury is uncommon. Similarly, the number of exposures, particularly those occurring in rapid succession, is difficult to document. Battlefield accounts suggest that repetitive exposure in rapid succession is commonplace, and the dose response of repetitive blast exposure in humans is unknown. Without baseline measurements, it is not possible to infer a causal relationship between blast exposure, alterations in WM integrity, and performance on neuropsychological tests with complete certainty. The selfreport and retrospective nature of TBI assessment and diagnosis also limits the certainty of group assignment. Finally, the resolution of the DTI scan was limited with a large spacing between slices ( $z$ dimension of $6 \mathrm{~mm}$ ) relative to the in-plane resolution $(2 \mathrm{~mm})$.

\section{CONCLUSION}

Blast-exposed veterans not reporting acute symptoms of TBI display WM abnormalities comparable to cases of primary blast-related mild TBI. Our results are consistent with recent reports of WM abnormalities from repetitive subconcussive events in elite sports participants, and suggest the DTI $z$ score analytic method may be quite sensitive to the diffuse and heterogeneousinjuries characteristic of such events. The lack of clear TBI symptoms following blast exposure may lead to the erroneous assumption that there has been little or no effect on the central nervous system. Such injuries may 
produce subtle cognitive deficits or later manifestation of symptoms. Our results must be considered preliminary and necessitate replication by using larger sample sizes to evaluate the clinical utility of DTI $z$ score analysis. If confirmed, our findings would argue for supplementing the established approach for making diagnoses based purely on clinical history and observable acute symptoms of mild TBI with novel imagingbased diagnostic criteria that "look below the surface" for pathology. ${ }^{14,15,44}$

\section{REFERENCES}

1. Bochicchio GV, Lumpkins $\mathrm{K}$, O’Connor J, et al. Blast injury in a civilian trauma setting is associated with a delay in diagnosis of traumatic brain injury. Am Surg. 2008;74:267-270.

2. Cernak I, Noble-Haeusslein L. Traumatic brain injury: an overview of pathobiology with emphasis on military populations. J Cereb Blood Flow Metab. 2010;30:255-266.

3. Chen Y, Huang W. Non-impact, blast-induced mild TBI and PTSD: concepts and caveats. Brain Inj. 2011;25:641-650.

4. MacGregor AJ, Dougherty AL, Galarneau MR. Injury-specific correlates of combat-related traumatic brain injury in Operation Iraqi Freedom. J Head Trauma Rehabil. 2011;26:312-318.

5. Bass CR, Panzer MB, Rafaels KA, Wood G, Shridharani J, Capehart B. Brain injuries from blast. Ann Biomed Eng. 2012;40:185-202.

6. Sams R, LaBrie DW, Norris J, Schauer J, Frantz E. IED blast postconcussive syncope and autonomic dysregulation. Mil Med. 2012;177:48-51.

7. Yeh DD, Schecter WP. Primary blast injuries-an updated concise review. World J Surg. 2012;36:966-972.

8. Duckworth JL, Grimes J, Ling GSF. Pathophysiology of battlefield associated traumatic brain injury. Pathophysiology. 2013;20: 23-30.

9. Ganpule S, Alai A, Plougonven E, Chandra N. Mechanics of blast loading on the head models in the study of traumatic brain injury using experimental and computational approaches. Biomech Model Mechanobiol. 2013;12:511-531.

10. Management of Concussion-mTBI Working Group. VA/DoD clinical practice guideline for management of concussion $/ \mathrm{mild}$ traumatic brain injury. J Rehabil Res Dev. 2009;46:CP1-CP68.

11. MacDonald C, Johnson A, Cooper D, et al. Cerebellar white matter abnormalities following primary blast injury in US military personnel. PLoS One. 2013;8:e55823.

12. Rosen A, Zhang Y, Zhan W, et al. Radiology corner. Case 41. Arcuate fasciculus damage seen on DTI in a blast-exposed soldier with mild traumatic brain injury (mTBI) with associated conduction aphasia. Mil Med. 2009;174:v-vi.

13. Hayes JP, Morey RA, Tupler LA. A case of frontal neuropsychological and neuroimaging signs following multiple primary-blast exposure. Neurocase. 2012;18:258-269.

14. Le Bihan D. Looking into the functional architecture of the brain with diffusion MRI. Nature Rev Neurosci. 2003;4:469-480.

15. Le Bihan D, Johansen-Berg H. Diffusion MRI at 25: exploring brain tissue structure and function. Neuroimage. 2012;61: 324-341.

16. Bazarian JJ, Donnelly K, Peterson DR, Warner GC, Zhu T, Zhong $\mathrm{J}$. The relation between posttraumatic stress disorder and mild traumatic brain injury acquired during Operations Enduring Freedom and Iraqi Freedom. J Head Trauma Rehabil. 2013;28:1-12.

17. Jensen KB, Kaptchuk TJ, Kirsch I, et al. Nonconscious activation of placebo and nocebo pain responses. Proc Natl Acad Sci US A. 2012;109:15959-15964.

18. Dashnaw ML, Petraglia AL, Bailes JE. An overview of the basic science of concussion and subconcussion: where we are and where we are going. Neurosurg Focus. 2012;33:E5.
19. Lipton ML, Kim N, Zimmerman ME, et al. Soccer heading is associated with white matter microstructural and cognitive abnormalities. Radiology. 2013;268:850-857.

20. Koerte IK, Ertl-Wagner B, Reiser M, Zafonte R, Shenton ME. White matter integrity in the brains of professional soccer players without a symptomatic concussion. JAMA. 2012;308: 1859-1861.

21. Bazarian JJ, Zhu T, Blyth B, Borrino A, Zhong J. Subject-specific changes in brain white matter on diffusion tensor imaging after sports-related concussion. Magn Reson Imaging. 2012;30:171-180.

22. Koerte IK, Kaufmann D, Hartl E, et al. A prospective study of physician-observed concussion during a varsity university hockey season: white matter integrity in ice hockey players. Part 3 of 4 . Neurosurg Focus. 2012;33:E3.

23. Breedlove EL, Robinson M, Talavage TM, et al. Biomechanical correlates of symptomatic and asymptomatic neurophysiological impairment in high school football. J Biomech. 2012;45:12651272 .

24. Talavage TM, Nauman EA, Breedlove EL, et al. Functionallydetected cognitive impairment in high school football players without clinically-diagnosed concussion [published online ahead of print April 11, 2013]. J Neurotrauma. doi: 10.1089/neu.2010.1512.

25. Goldstein LE, Fisher AM, Tagge CA, et al. Chronic traumatic encephalopathy in blast-exposed military veterans and a blast neurotrauma mouse model. Sci Transl Med. 2012;4:134ra60.

26. Kennedy PE. Randomization tests in econometrics. JBus Econ Stat. 1995;13:85-94.

27. Anderson MJ, Robinson J. Permutation tests for linear models. Aust N Z J Stat. 2001;43:75-88.

28. Smith SM, Nichols TE. Threshold-free cluster enhancement: addressing problems of smoothing, threshold dependence and localization in cluster inference. Neuroimage. 2009;44:83-98.

29. Cubon VA, Putukian M, Boyer C, Dettwiler A. A diffusion tensor imaging study on the white matter skeleton in individuals with sports-related concussion. J Neurotrauma. 2011;28:189-201.

30. Messe A, Caplain S, Paradot G, et al. Diffusion tensor imaging and white matter lesions at the subacute stage in mild traumatic brain injury with persistent neurobehavioral impairment. Hum Brain Mapp. 2011;32:999-1011.

31. Zhuang L, Wen W, Zhu W, et al. White matter integrity in mild cognitive impairment: a tract-based spatial statistics study. Neuroimage. 2010;53:16-25.

32. Mori S, Wakana W, Nagae-Poetscher LM, Van Zijl PCM. MRI Atlas of Human White Matter. 1st ed. Amsterdam, the Netherlands: Elsevier; 2005.

33. Lilliefors HW. On Kolmogorov-Smirnov test for normality with mean and variance unknown. J Am Stat Assoc. 1967;62:399-402.

34. Jorge RE, Acion L, White T, et al. White matter abnormalities in veterans with mild traumatic brain injury. Am J Psychiatry. 2012;169:1284-1291.

35. White T, Ehrlich S, Ho B-C, et al. Spatial characteristics of white matter abnormalities in schizophrenia. Schizophr Bull. 2013;39:1077-1086. 
36. Schuff N, Zhang Y, Zhan W, et al. Patterns of altered cortical perfusion and diminished subcortical integrity in posttraumatic stress disorder: a MRI study. Neuroimage. 2011;54:S62-S68.

37. Yeh PH, Simpson K, Durazzo TC, Gazdzinski S, Meyerhoff DJ. Tract-based spatial statistics (TBSS) of diffusion tensor imaging data in alcohol dependence: abnormalities of the motivational neurocircuity. Psychiatry Res. 2009;173:22-30.

38. Madden DJ, Whiting WL, Huettel SA, White LE, MacFall JR, Provenzale JM. Diffusion tensor imaging of adult age differences in cerebral white matter: relation to response time. Neuroimage. 2004;21:1174-1181.

39. Bigler ED, Maxwell WL. Neuropathology of mild traumatic brain injury: relationship to neuroimaging findings. Brain Imaging Behav. 2012;6:108-136.

40. Shenton ME, Hamoda HM, Schneiderman JS, et al. A review of magnetic resonance imaging and diffusion tensor imaging findings in mild traumatic brain injury. Brain Imaging Behav. 2012;6:137192.

41. Blyth BJ, Bazarian JJ. Traumatic alterations in consciousness: traumatic brain injury. Emerg Med Clin N Am. 2010;28: 571-594.

42. Buki A, Povlishock JT. All roads lead to disconnection?traumatic axonal injury revisited. Acta Neurochir (Wien). 2006;148: 181-193.

43. Farkas O, Povlishock JT. Cellular and subcellular change evoked by diffuse traumatic brain injury: a complex web of change extending far beyond focal damage. Prog Brain Res. 2007;161:43-59.
44. Song SK, Sun SW, Ramsbottom MJ, Chang C, Russell J, Cross AH. Dysmyelination revealed through MRI as increased radial (but unchanged axial) diffusion of water. Neuroimage. 2002;17:14291436.

45. Pierpaoli C, Barnett A, Pajevic S, et al. Water diffusion changes in Wallerian degeneration and their dependence on white matter architecture. Neuroimage. 2001;13:1174-1185.

46. Wheeler-Kingshott CA, Cercignani M. About "axial” and "radial” diffusivities. Magn Reson Med. 2009;61:1255-1260.

47. Jones DK. Determining and visualizing uncertainty in estimates of fiber orientation from diffusion tensor MRI. Magn Reson Med. 2003;49:7-12.

48. Wedeen VJ, Rosene DL, Wang R, et al. The geometric structure of brain fiber pathways. Science. 2012;335:1628-1634.

49. Fakhran S, Yaeger K, Alhilali L. Symptomatic white matter changes in mild traumatic brain injury resemble pathologic features of early Alzheimer dementia. Radiology. 2013;269:249-257.

50. Sorg SF, Delano-Wood L, Luc N, et al. White matter integrity in veterans with mild traumatic brain injury: associations with executive function and loss of consciousness. J Head Trauma Rehabil. 2014;29:21-32.

51. Bartres-Faz D, Arenaza-Urquijo EM. Structural and functional imaging correlates of cognitive and brain reserve hypotheses in healthy and pathological aging. Brain Topogr. 2011;24:340-357.

52. Moretti L, Cristofori I, Weaver SM, Chau A, Portelli JN, Grafman J. Cognitive decline in older adults with a history of traumatic brain injury. Lancet Neurol. 2012;11:1103-1112. 